\title{
The Italian Consensus Conference on Pain in Neurorehabilitation
}

Maria Gabriella Buzzi ${ }^{1}$, Stefano Tamburin ${ }^{2}$, Giorgio Sandrini ${ }^{3}$

IRCCS Fondazione Santa Lucia, Rome, Italy

Department of Neurological and Movement Sciences, University of Verona, Italy

C. Mondino National Institute of Neurology Foundation, IRCCS, Department of Brain and Behavioural Sciences,

University of Pavia, Pavia, Italy

\section{Introduction}

Pain is frequent in several neurological conditions, such as stroke, Parkinson's disease, multiple sclerosis, diabetic neuropathy, but it is often underestimated and therefore untreated or not successfully treated. Pain reduces quality of life, it may be the cause of adaptive disorders (such as anxiety and depression) and, in patients undergoing rehabilitation, it may negatively impact rehabilitation procedures and hamper the outcome. Unfortunately, there are no significant data regarding the impact of pain in neurorehabilitation patients and what would be the impact of an appropriate pain treatment on rehabilitation outcome. Accordingly, there are no guidelines providing indications on how to treat pain in patients with pain during rehabilitation treatment and thereafter. Given this background, and to comply with the Italian law 38 released on 2010 by the Italian Ministry of Health to guarantee adequate care and treatment of patients with pain both in hospital and in primary care, the Italian Society for Neurological Rehabilitation (SIRN) along with the Italian Society for Physical and Rehabilitative Medicine (SIMFER), promoted a work-in-progress platform, the Italian Consensus Conference on Pain in Neurorehabilitation (ICCPNR).

\section{The Italian Consensus Conference on}

\section{Pain in Neurorehabilitation}

The ICCPNR involved several scientific societies interested in pain and/or neurological diseases and aimed to provide recommendations for the evaluation, diagnosis and cure of pain in patients undergoing neurorehabilitation. 


\section{General issues groups}

- Translation research on pain

- Neuropathic, nociceptive and mixed pain: differential diagnosis

- Instrumental evaluation of pain

- Sex-related pain biomarkers

- The psychiatric co-morbidity and the anthropological and cultural dimensions of pain

- The psychological dimension of pain

- Pharmacological treatment of pain, including opioids and invasive procedures

- Physical and complementary therapies for pain

- Coping strategies, psychotherapy and cognitivebehavioural therapies for pain

- The role of nurses, physical therapists, and occupational therapists

Table I. General issues groups

\section{Specific diseases groups}

- Pain and spasticity

- Pain in acute and chronic stroke

- Pain in multiple sclerosis

- Pain in spinal cord injury

- Pain in cerebral palsy and in pediatric patients

- Pain in movement disorders

- Pain in motor neuron diseas

- Pain in chronic disorders of consciousness and dementia

- Pain in oncology and neuro-oncology

- Pain in neuro-infettivology

- Pain in plexopathy, radiculopathy and mononeuropathy

- Deafferentation and phantom limb pain

- Pain in peripheral neuropathies

- Headache and facial pain

- Low back pain and failed back surgery syndrome

- Osteoarticular pain

- Myofascial pain and fibromyalgia

- Chronic pelvic pain

Table II. Specific diseases groups pain and its care. The final document will be published as a supplement of an international peerreviewed journal

The ICCPNR is an useful instrument to reach, trough a formal process, an agreement on controversial and complex issues, to favour homogeneous approaches in clinical practice. The final document will represent an useful reference in case of uncertainty on specific issues or for topics which have not and/or cannot be dealt with quantitative studies.

The ICCPNR is composed by the Promoter Committee, the Technical-Scientific Committee, the Working Groups, and the Jury. The ICCPNR start-up meeting was held on march 2012, the panel is divided into 10 groups dealing with general issues and 18 groups dealing with specific diseases (Table I).

Each group dealt with specific questions to define the level of evidence of currently published literature and to grade recommendation according to international guidelines. The systematic review of literature was done through launching keywords on the specific questions in the following databases: PubMed, Medline, Embase, The Cochrane Library.

The draft of the answers to questions, and of the recommendation proposals will be reviewed by an interdisciplinary jury representing the different possible approaches to any specific topic. The jury will draw final conclusion on the recommendations.

\section{Conclusions}

The work of the ICCPNR will represent the basis for better recognition, evaluation and treatment of pain in neurorehabilitation. It will also represent a starting point for better application of the Italian law 38/2010 regarding 